\author{
Monika Anna Noga \\ Uniwersytet we Fryburgu \\ monika.anna.noga@gmail.com
}

\title{
Zum Topos des „Gärtners” bei Leopold Staff (Ogrodnicy)
}

\section{On the Topos of the Gardener in Leopold Staff (Ogrodnicy)}

\begin{abstract}
The article discusses in detail a poem by Leopold Staff entitled Ogrodnicy [Gardeners]. The main object of the hermeneutic and structural analysis of this poem is the "topos of the gardener." It appears in writings of authors strongly influenced by the classical tradition, and Staff was a Neoclassicist. The poem Ogrodnicy can be read as a text from this tradition. Its analysis is meant to characterize the topos of the gardener and point at the role of a modern poet and modern Polish literature.
\end{abstract}

Keywords: Leopold Staff, topos, topos of the gardener, garden, neoclassicism.

Streszczenie: W artykule szczegółowo omówiony został wiersz Leopolda Staffa Ogrodnicy. Główny przedmiot analizy hermeneutyczno-strukturalnej tego utworu stanowi „topos ogrodnika”. Występuje on u autorów, którzy są pod silnym wpływem tradycji klasycznej, a Staff należał właśnie do neoklasycystów. Wiersz Ogrodnicy może być odczytany jako tekst tej tradycji. Analiza wiersza ma pokazać specyfikę „toposu ogrodnika” oraz wskazać rolę współczesnego poety i współczesnej literatury polskiej.

Słowa kluczowe: Leopold Staff, topos, topos ogrodnika, ogród, neoklasycyzm

Przez ogród mój szatan szedł smutny śmiertelnie I zmienił go w straszną, okropną pustelnię...

$\mathrm{Z}$ ponurym, na piersi zwieszonym szedł czołem

I kwiaty kwitnące przysypał popiołem,

Trawniki zarzucił bryłami kamienia

I posiał szał trwogi i śmierć przerażenia...

Aż, strwożon swym dziełem, brzemieniem ołowiu

Położył się na tym kamiennym pustkowiu, 
By w piersi łkające przytłumić rozpacze,

I smutków potwornych płomienne łzy płacze... ${ }^{1}$

Der folgende Text stammt aus dem Gedicht Deszcz jesienny von Leopold Staff aus dem Gedichtband Sny o potędze (1901, Mächtigkeitsträume), mit dem Staff als typischer Vertreter des Jungen Polen debütierte. Staff wird als Dichter von drei Generationen ${ }^{2}$ bezeichnet. Er ist in drei Literaturepochen eingeordnet worden: Junges Polen, Zwischenkriegszeit und Nachkriegszeit.

In jeder dieser drei Epochen galt er als Autorität und Vorbild ${ }^{3}$ für junge Autoren ${ }^{4}$, und in jeder Phase seiner Arbeit ist er immer als ein moderner und universeller Dichter ${ }^{5}$ betrachtet worden. Jerzy Kwiatkowski schreibt:

Das poetische Wirken des Leopold Staff stellt ein erstaunliches und in diesem Ausmaß in der polnischen Literatur ein einzigartiges Beispiel für die Langlebigkeit und Regenerationsfähigkeit von Poesie dar (...). Staff ist der Mitbegründer einiger literarischer Phasen, welche durch Literaturhistoriker auch gerne Epochen genannt werden. In jeder dieser Epochen spielt Staff eine Hauptrolle ${ }^{6}$.

Das zitierte Fragment ist zugleich das Finalbild dieses Werkes. Den Garten des Dichters kann man metaphorisch als das verlorene Paradies interpretieren und die „flammenden Tränen” tens durch den Satan. „Płomienne łzy”: Dieses Weinen muss nicht nur die Trauer über Geschehenes bedeuten, sondern kann auch als fortdauernde Zerstörung der Welt gesehen werden, die aufzuhalten, niemand in der Lage ist: Nun regnet es die ganze Zeit.

Wenn Staff also im Jahr 1903 verkündet, in seinem Garten sei der Satan gewesen, habe die Welt in eine Wüste verwandelt und alles um sich herum zerstört, bedeutet das dann auch das Ende des Entstehens von anderen Gärten und dadurch das Ende der Funktion des Gärtners bzw. von Gärtnern bei Staff?

Der Begriff des Gartens eignet sich gut für eine vielseitige topische Verwendung, weil seit der Schöpfungsgeschichte der Bibel bis unsere Zeit in vielen Kulturen mit Assoziationen belegt werden kann.

${ }^{1}$ L. Staff, Poezje zebrane, t. 1, Warszawa 1967, s. 299-300. Band Dzień duszy aus dem Jahr 1903.

${ }^{2}$ L. Staff [in:] A. Hutnikiewicz, Mtoda Polska, Warszawa 2004, s. 124.

${ }^{3}$ In der Zwischenkriegszeit hat er einen großen Einfluss auf die Skamandriten ausgeübt. J. Kwiatkowski, Literatura Dwudziestolecia, Warszawa 1990, s. 89.

${ }^{4}$ M. Jastrun, Wstęp [in:] L. Staff, Wybór poezji, Wrocław 1970, s. XXXVI.

${ }^{5}$ L. Staff [in:] A. Hutnikiewicz, op. cit., s. 124, 127.

${ }^{6}$ „Poezja Leopolda Staffa stanowi - w tej skali jedyny w polskiej literaturze - przykład zdumiewającej długowieczności i zdumiewających zdolności regeneracyjnych. (...) Staff jest współtwórcą paru okresów literackich, chętnie przez historyków literatury zwanych epokami. W każdej z nich - odgrywa rolę pierwszorzędną".

7 Übers. von M.A.N. 
Dies gilt für das 1954 veröffentlichte Gedicht Ogrodnicy von Leopold Staff (1878-1957).

Die zentrale Themenstellung ist die Modellierung des Topos des

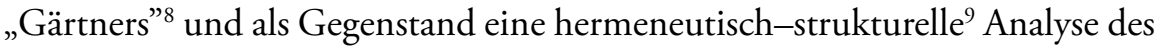
erwähnten Gedichtes.

Die Textanalyse soll die Eigenart des Gärtner-Topos aufzeigen, und was der Text über den Topos des „Gärtners”, die Rolle des modernen Dichters und der modernen Literatur allgemein verrät.

Das Gedicht Staffs, Ogrodnicy, wurde drei Jahre vor seinem Tod (er starb im Alter 76 Jahren) 1954 publiziert, d.h. der Dichter schuf das lyrische Werk in der letzten Phase des Lebens. Ist das eine Art Abschiedsbriefes? Ein Testament? Ein Gewissen Rechnung?

Oder ganz im Gegenteil, ist das eine Art Lebensverlängerung nach ihrem Tod? Wie Janusz Kofta über die Gärten gesungen hat:

Pamiętajcie o ogrodach

Przecież stamtąd przyszliście ${ }^{10}$.

${ }^{8}$ In der Literaturwissenschaft konzentriert sich das Verständnis des Begriffes „Topos” seit den Forschungsergebnissen von Ernst Robert Curtius (1948, Europäische Literatur und lateinisches Mittelalter) auf ähnliche Beschreibungen. Hier ist von „festen Klischees oder Denk- und Ausdrucksschemata" die Rede, welche ebenso Zitate, Bilder und Motive umfassen können; der methodische Ansatz ist ahistorisch.

Rainer Grübel dagegen versucht den Toposbegriff um die zeitliche und räumliche Komponente zu erweitern. Als Ansatz hierzu geht er von den Verhältnissen aus, die Phänomene zueinander einnehmen können und unterteilt jene in zwei Kategorien: Das zeitliche Verhältnis von Phänomenen zueinander, welches er als chronische Modellierung beschreibt, sowie das räumliche Verhältnis von Phänomenen zueinander, welches die topische Modellierung ist. (R. Grübel, Zwischen "Leier" und "Trommel”, zur Funktion zweier Topoi im Wechselverhältnis von Struktur und Selbstverständnis russischer Avantgardistischer Lyrik, „Wiener Slawistischer Almanach”, red. AA. Hansen-Löve, Bd. 2, Wien 1978, s. 25). Laut Grübel „Ordnet” die topische Modellierung „die Phänomene nebeneinander an”, während die chronische Modellierung sie nacheinander anordnet „(diatopisch vs. diachronisch); sie können desweiteren als gleichzeitig bzw. gleichräumig (synchronisch vs. isotopisch), als immerwährend bzw. überall vorhanden (panchronisch vs. pantopisch) und schließlich als nie bzw. nirgendwo anzutreffend (urchronisch vs. utopisch) modelliert werden”. Der Begriff „Topos” findet sich in einschlägigen (deutschsprachigen und polnischen) Literaturlexika. Den Begriff „Topos des Gärtners” allerdings sucht man in den Enzyklopädien vergeblich.

9 "Jeder Text hat also vier Dimensionen - die historische (...), die technische (linguistische) (...), die ästhetische (philosophische) (...) und die kulturelle (...). Die ästhetische Dimension „Warum” bezieht sich hierbei auf den sensus anagogicus (der symbolische Sinn), der allegorische Sinn, sensus allegoricus, auf das kulturelle „Warum”. Der buchstäbliche Sinn, sensus litteralis, meint das historische „Warum”. Der tropologische Sinn, sensus moralis, entspricht technischen "Warum”. M. Freise, Vier Weisen, nach dem Text zu fragen [in:] Finis coronat opus. Festschrift für Walter Kroll zum 65. Geburtstag, Göttingen 2006, s. 71-83.

${ }^{10}$ J. Kofta, Pamiętajcie o ogrodach, http://www.tekstowo.pl/piosenka,jonasz_kofta, pamietajcie_o_ogrodach.html, abgerufen am 24.07.2011. 


\section{Leopold Staff Ogrodnicy ${ }^{11}$}

51 Jahre später ließ sich der Autor mit sich selbst auf eine Diskussion über das Gedicht Deszcz jesienny ein. Er schrieb ein neues Gedicht - Ogrodnicy. Der Titel selbst deutet, dass der Dichter beim Thema „Garten” bleibt. Die Frage ist: bekräftigt er immer wieder seine früheren Äusserungen oder er trennt sich von ihnen?

Bei dieser Analyse geht es in erster Linie nicht darum, reale Ereignisse zu rekonstruieren. Es ist jedoch sinnvoll, den Entstehungskontext des Bands Wiklina (1954, Weidenruten), der für den Text relevant ist, kurz zu skizzieren.

\section{Historisches Warum}

Das Gedicht Ogrodnicy stammt aus dem Jahre 1954. Dieses Datum deutet darauf hin, dass das Werk während der Nachkriegszeit entstanden ist. Nach dem Wörterbuch der polnischen Sprache ${ }^{12}$ ist „wiklina” (Weidenrute) eine „Weidenart mit roten jungen Trieben, langen grau-grünen Blättern, die am Ufer von Flüssen ein Dickicht bilden"13. Sie wird geflochten und so im Korbhandwerk für die Herstellung von Körben und anderen Dekorationen verwendet.

In den Denkschriften des Priesters Antoni Boratyński (Wspominam Leopolda Staffa) wird erwähnt, dass Staff die meisten Gedichte aus dem Wiklina Band bereits im Sommer 1953 schrieb. In diesem Sommer verbrachte die Familie Staff ihre Sommerferien im Pfarrhaus in Skarżysko-Kamienna. Boratyński schreibt:

Przemiły to był czas gawęd, wspomnień, spacerów po łąkach i ogrodzie plebańskim (...). Tuż za ogrodem płynie rzeka Kamienna i jej niewielki dopływ Bernardka; brzegi ich są bujnie porośnięte wikliną. Stąd tytuł tomu poezji z r. 1954: Wiklina ${ }^{14}$.

Allerdings ist in einem Interview, das am 22. Januar 1953 veröffentlicht wurde, zu lesen, dass der geplante Gedichtband den Titel Zmarszczki na wo$d z i e$ tragen sollte. In einem anderen Interview für die Zeitung „Życie Warszawy" vom 15. November 1953 sagt Staff aber:

${ }_{11}$ M. Szypowska, Leopold Staff. Wiersze zebrane, t. 5, Warszawa 1955, s. 352.

12 Stownik języka polskiego, red. M. Szymczak, Warszawa 1978, s. 716.

13 Übers. von M.A.N.

${ }_{14}$ A. Boratyński, Wspominam Leopolda Staffa, http://www.skarzysko24.pl/content/ view/748/109/, abgerufen am 24.08.2011. „Es war eine sehr angenehme Zeit der Plaudereien, der Erinnerungen, der Spaziergänge auf den Wiesen und im Pfarrhausgarten (...). Gleich hinter dem Garten fließt der Fluss Kamienna und sein kleiner Nebenfluss Bernardka; ihre Ufer sind üppig mit Weidenruten bewachsen. Daher auch der Titel des Gedichtbandes aus dem Jahr 1954: Weidenruten" [übers. von M.A.N.]. 
Ukończyłem właśnie tom nowych wierszy dla Państwowego Instytutu Wydawniczego. Wiersze te mają już wkrótce ukazać się na półkach księgarskich, ale nie wiadomo jeszcze pod jakim tytułem. Ja osobiście zaproponowałem tytuł Wiklina ${ }^{15}$.

Staff hat mit der Wahl des passenden Titels offensichtlich gezögert. Den im Verlag festgelegte Titel lehnte er ab. Laut Irena Maciejewska war der neue Titel „mit seiner Einfachheit, Bescheidenheit und mit seinen (...) metaphorischen Bedeutungen verlockender" ${ }^{16}$. So kommentiert dies der Autor selbst im weiteren Teil des zitierten Interviews wie folgt:

dlatego że wiklina bez cudzysłowu to taka sobie skromna roślinka, takie sobie niepozorne pręciki, z których, można powiedzieć żartobliwie, muza uplotła dla mnie kosz. Kosz, który od niej dostałem. (...)

- Jeśli już twierdzi pan, iż muza dała mu kosz, to chyba był to kosz pełen ${ }^{17}$.

- Pełen, pełen - śmieje się Staff. - Nawet bardzo pełen. Dźwigam go już tyle

lat, a on ciągle jest pełen i ciężki. No, bo proszę, dopiero co skończyłem któryś tam $\mathrm{z}$ rzędu tom wierszy, a tu już czuję w koszu nowy ciężar ${ }^{18}$.

In dem Interview sagt Staff, die Muse als Göttin der Kunst habe für ihn einen Korb geflochten, der sich wie das Füllhorn von Almatheia immer wieder mit neuen Werken füllt. Im übertragenen Sinne ist das Weben und Flechten von Wörtern als das Entstehen eines (literarischen) Werkes zu verstehen. Das Gedicht bekommt damit eine bewusst arrangierte Konstruktion. Der Künstler versteht sich als ein Handwerker, der mit Wortmaterial arbeitet.

W parku łagodni ogrodnicy

Rozpylaczami koszą grządki.

15 A. Wysocki, Poeta nescit finire. U Leopolda Staffa (w 75-lecie urodzin), „Życie Warszawy” 1953, nr 273. „Ich habe gerade einen neuen Gedichtband für den Verlag Państwowy Instytut Wydawniczy vollendet. Diese Gedichte werden bald in den Buchhandlungsregalen liegen, es ist aber noch nicht klar, mit welchem Titel. Persönlich schlug ich den Titel Weideruten vor" [übers. von M.A.N.].

${ }^{16}$ Ibidem, s. 397-398.

17 Das Adjektiv „pełen” bedeutet hier 'ausgefühlt mit dichterischer Inspiration'; aus dem vollen Korb (aus Weidenruten) holt sich Staff neue Ideen für sein dichterisches Schaffen.

18 Zitiert nach: I. Maciejewska, Leopold Staff. Warszawski okres twórczości, Warszawa 1973, s. 397-398, „weil Weiderute ohne Anführungszeichen eine so schlichte kleine Pflanze ist, die so unscheinbare Staublätter hat, aus welchen, scherzhaft gesagt, die Muse für mich einen Korb geflochten hat. Diesen Korb habe ich von ihr erhalten (...).

- Wenn Sie bereits gesagt haben, dass die Muse Ihnen einen Korb geschenkt hat, bedeutet das wahrscheinlich einen vollen Korb

- Voll, voll - lacht Staff - Sogar ganz voll. Ich schleppe ihn bereits so viele Jahre und er ist immer noch voll und schwer. Nun, bitte schön, ich habe gerade den soundsovielten Gedichtband beendet und nun spüre ich bereits eine neue Last im Korb” [übers. von M.A.N.]. 


\section{Technisches Warum}

Das Wortmaterial des Gedichts Ogrodnicy von Leopold Staff ist in drei Strophen unterteilt. Jede Strophe besitzt eine gerade Anzahl von Versen. Die erste Strophe besteht aus acht Versen, die zweite aus vier und die dritte und letzte wieder aus acht Versen. Somit ist der Text ganzzahlig, basierend auf der Zahl vier in $2 \times 4+1 \times 4+2 \times 4$ Versen segmentiert. Außerdem besteht das gesamte Gedicht aus acht Sätzen. Es liegt also nahe, dass diese Zahl eine spezielle Funktion erfüllt. Die absichtliche, grafisch-harmonische Bauart des Textes realisiert die Form des Themas. Sie zeigt einen Ort, in diesem Fall die Struktur eines gut organisierten Gartens.

Bei Ogrodnicy handelt es sich um ein tonisches Gedicht ${ }^{19}$, in dem drei Akzenteinheiten dominieren:

1 W parku lagodni ogrodnicy

2 Rozpylaczami koszą grządki

3 Hiacyntów i storczyków.

4 Taka jest moc nawyku! $!^{20}$

5 Bo pomną, jak to ongi

6 Kropili z lepszej broni

7 W kamienne koronki

8 Gotyckich wież i wieżyczek.

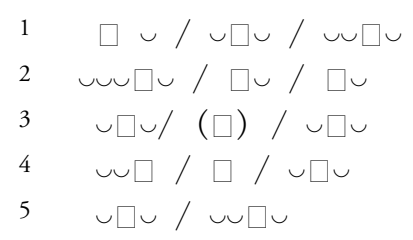

${ }_{19}$ Das tonische Verssystem ist eine populäre Versform des Modernismus. Als Manifest des polnischen Tonismus gilt das Werk Ksiega ubogich (Das Buch der Armen) von Jan Kasprowicz.

Im tonischen System dieses Gedichts gibt es keine regelmäßige Folge von Akzenten (wie im Falle der syllabotonischen Poesie) und keine gleiche Anzahl an Silben in den Versen (wie bei syllabischen Gedichten). In jedem Vers befinden sich 7 bis 10 Silben. Es lassen sich Silbenakzente feststellen. In der Regel finden sich in diesem Gedicht pro Vers drei Silbenakzente (wie es im polnischen Tonismus am häufigsten der Fall ist). Bisweilen finden sich Verse mit vier Silbenakzenten. So ähneln die Akzente im 10. Vers einem jambischen Rhythmus, vier Versfüßen, in denen jeweils die erste Silbe unbetont, die zweite betont ist. Das wäre ein jambischer, katalektischer Vers mit vier Silbenakzenten und einer schwachen (= unbetonten) Silbe am Ende. Dies wiederum hängt mit dem paroxytonischen Akzent zusammen (mit der regelmäßig auf der vorletzten Silbe liegenden Betonung des Polnischen).

${ }^{20}$ Laut Maria Dłuska ist auch die Art und Weise, wie man mit der Stimme ein Gedicht interpretiert, bringt den tonischen Charakter des Gedichtes zur Geltung. Einige Akzente sind fakultativ eingesetzt und kommen erst bei der Rezitation zum Vorschein. Vgl. M. Dłuska, Prozodia języka polskiego, Kraków 1947, s. 51-53. 


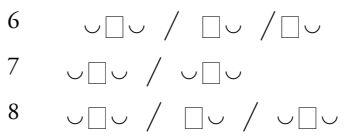

Das tonische System wird einerseits von der gleichen Anzahl der Akzentsilben (der Versfüße) charakterisiert, andererseits fordert es eine unregelmäßige Verteilung von Akzente. Dies lässt sich mit der räumlichen Organisation eines Gartens vergleichen. Einerseits richtet ein Gärtner seinen Garten ein und pflegt ihn, andererseits lässt er der ursprünglichen, wild wuchernden Natur eine gewisse Freiheit. Das Kreative des Autors besteht also in der grafischen Gliederung und Planung des Textes sowie in seiner individuellen sprachlichen Interpretation $^{21}$, die der Arbeit eines Gärtners ähnelt, der seinen Garten plant und pflegt. Dieser Garten symbolisiert die Beherrschung der Natur durch den Menschen „und die Macht der Vernunft über die Impulse des Unterbewusstseins"22. In ähnlicher Weise muss der Dichter seine Worte zähmen.

Die sprechende Person im Gedicht ist ein lyrisches „Ich” männlichen Geschlechts. Darauf lässt die männliche Verbform „szedłem” schließen. Es zeigt seine Anwesenheit erst im kürzesten Fragment des Gedichtes, vor allem im mittleren Teil, d.h. in der vierzeiligen Strophe, die einen Reim nach dem Schema 'abba' besitzt, während die anderen Strophen keine Reime besitzen. In der ersten Strophe ist das lyrische „Ich” ein beschreibender, distanzierter Beobachter, der die Gedanken der von ihm beobachteten Gärtner durchschauen und lesen kann.

Beim Lesen des Gedichtes bemerkt man Begriffe die aufgrund der Assoziationen mit denen sie beladen sind, jeder Strophe einen bestimmten Abschnitt der polnischen (Literatur) Geschichte zuordnen. Man kann die Abschnitte folgendermaßen deutend zusammenfassen ${ }^{23}$ :

1. Strophe - Avantgarde

2. Strophe -1 . und 2. Weltkrieg

3. Strophe - Junges Polen.

${ }^{21}$ Vgl. ibidem.

${ }^{22}$ Vgl. M. Battistini, Symbole i alegorie, tłum. K. Dyjas, Warszawa 2005, s. 252. 23

\begin{tabular}{|c|c|c|}
\hline 1. Strophe & 2. Strophe & 3. Strophe \\
\hline W parku łagodni ogrodnicy & Od tych dni dzieli mnie już & Tymczasem nawodniona Sahara \\
\hline Rozpylaczami koszą grządki & wiele drzew, & Rozkwitła cała jak jeden sad. \\
\hline Hiacyntów i storczyków. & Wśród których szedłem przez & W nocy księżyc podlewa go rosą \\
\hline Taka jest moc nawyku! & ogrody, & I w mgle tancerka tańczy boso, \\
\hline Bo pomną, jak to ongi & I upłynęło tyle wody, & Wśród kwiatów sama jak kwiat. \\
\hline Kropili z lepszej broni & Jakby to była ludzka krew. & We dnie, pod gołym niebem goły, \\
\hline W kamienne koronki & & W słońcu opala się mój brat. \\
\hline Gotyckich wież i wieżyczek. & & Ale ma trupa oczodoły. \\
\hline
\end{tabular}


Wie bereits gezeigt wurde, steht die erste Strophe in einem Verhältnis zur dritten. Daraus ergibt sich eine Gegenüberstellung von Avantgarde und Jungem Polen. Es stellt sich die Frage: Was gehört zur Poetik der Avantgarde, und was zur Poetik des Jungen Polen? Im weiteren Verlauf dieser Untersuchung wird diese Frage zu berücksichtigen sein.

Die thematische Unterteilung kommt nicht nur im Kontext des gesamten Textes oder im Zusammenhang mit den beiden achtzeiligen Strophen zum Ausdruck, sondern auch innerhalb der ersten Strophe. Die ersten vier Verse beziehen sich nämlich auf die Gegenwart. Sie schaffen das erste thematische Bild. Die nächsten vier Verse enthalten das zweite Bild, das sich auf das Vergangene bezieht, was die Wörter „pomną” und „ongi”24 betonen. An die Vergangenheit erinnern hier auch die gotischen Türme, im Stil der europäischen Architektur des späten Mittelalters erbaute Bauwerke. Diese Erinnerung in der Form des Schlafes oder eines Traums, die in der zweiten Hälfte der ersten Strophe auftaucht, enthält Elemente einer Reise und stellt eine Dimension dar, die zwar parallel zur Realität existiert, aber sehr weit von ihr entfernt ist. Die Person oder Personen, die diese zeitlichen Dimensionen verbinden, sind immer noch dieselben Gärtner.

\section{Wie entfaltet sich die Komposition?}

Um die Entwicklung der Komposition zu verstehen, muss man mit dem zweiten Teil der ersten Strophe anfangen:

Bo pomną, jak to ongi

Kropili z lepszej broni

W kamienne koronki

Gotyckich wież i wieżyczek.

Ein besonders bedeutendes Wort in diesem Abschnitt ist die Verbform „kropili”. Mehrere Bedeutungen verstecken sich hinter dem Infinitiv „kropić". Die erste Assoziation bezieht sich auf den Weihwasserwedel, welchen man in der Kirche - u.a. beim Segnen der Speisen - mit Weihwasser verwendet. Diese Assoziation verweist also auf den religiösen Kontext. Wenn man sich jedoch auf die lexikalische Definition stützt, so gelangt man zu folgenden Bedeutungsdimensionen:

${ }^{24}$ Bereits am Anfang des zwanzigsten Jahrhunderts hat Staff einige Archaismen wie „pomną” oder „ongi” verwendet. 
1. mit einer Flüssigkeit in Form von Tropfen begießen, besprengen;

2. in Bezug auf Regen: nicht besonders intensiv regnen;

3. umgangssprachlich: a) schlagen; b) schießen ${ }^{25}$.

Das Schlüsselwort des Gedichts ist in diesem Fall relativ ${ }^{26}$. Im Zusammengang mit dem zitierten Fragment, insbesondere in Zusammenhang mit dem Wort „broń”, kann man diesem Verb die dritte der oben vorgebrachten Bedeutungen zuschreiben. Dadurch erhält es eine Dimension, die vielleicht nicht besonders historisch exakt wirkt, die sich jedoch stark auf die Vergangenheit bezieht. Diese Beobachtung wird im weiteren Teil dieser Arbeit noch genauer erklärt werden.

Das Verb „kropić” verbindet sich mit einer Präpositionalphrase, die um ein Epitheton erweitert ist. Es bestimmt die Nominalphrase in dieser Wortgruppe. Diese Bezeichnung des Substantivs, die eine Komparativform („lepszy”) des Adjektivs „dobry” ist, weist auf eine Art des Vergleichs hin. „Z lepszej” fordert nämlich die Ergänzung/Erweiterung „lepszej niż” oder „lepszej od (czegoś/(kogoś)" und in diesem Fall bezieht es sich auf die in den ersten vier Strophen des Gedichts erinnerten Aktivitäten:

W parku łagodni ogrodnicy

Rozpylaczami koszą grządki

Hiacyntów i storczyków.

Taka jest moc nawyku!

d.h. auf die Arbeit der Titelhelden an den Orchideen und Hyazinthen. Auf diese Weise entsteht zwischen den beiden Aktivitäten „kosić” und „kropić” eine Äquivalenzbeziehung. Es geht hier nicht nur um die Komparation des Adjektivs, sondern auch gleichzeitig um die Verbindung zwischen den beiden Teilen des Gedichts. Es geht darum die innere Spannung zwischen ihnen zu zeigen. Die Komparativform eröffnet auch die Diskussion über die Ungleichheit bzw. über die Unterschiede zwischen den Gärtnern. Das führt zu einer Suche nach Eigenschaften, die sowohl für die heutigen, als auch für die damaligen „biblischen” Gärtner charakteristisch sind. Gleichzeitig zeigt es eine innere Zerrissenheit. Die Seelen der Gärtner befinden sich in einem Zwiespalt zwischen Gegenwart und Vergangenheit. Um genau diesen Zwiespalt geht es Staff. Es ist viel bedeutsamer, die innere Seite der Gärtner zu zeigen und die

25 Stownik jezyka polskiego, red. M. Szymczak, op. cit., s. 1052.

${ }^{26}$ Hier zeigen sich die Auswirkungen des militärischen Jargons auf die Sprache von Staff„kropič” ähnlich wie „kosić” bedeutet „mit leichteren Waffen zu schießen”. Vgl. Stownik jezzyka polskiego, red. J. Karłowicz, A.A. Kryński, W. Niedźwiedzki, t. 2, Warszawa 1900-1927, s. 562; Stownik jezzka polskiego, red. W. Doroszewski, t. 3, Warszawa 1961, s. 1036, 1154. 
menschliche Seele genauer zu betrachten, als zu zeigen, welche Waffe - ironischerweise - nützlicher ist.

Darüber hinaus gibt es eine ähnliche Beziehung zwischen den Verben, die in einem Verhältnis zueinanderstehen. Beide beginnen mit dem gleichen Buchstaben, mit „k”: „kropili” und „koszą”. Diese Äquivalenz wird noch im nächsten Absatz besprochen werden, da sie für den weiteren Verlauf der Untersuchung von Bedeutung ist.

Die nächsten vier Verse - das Herz des Gedichtes - bilden im Mittelteil einen Übergang zwischen der ersten und der letzten Strophe.

Od tych dni dzieli mnie już wiele drzew,

Wśród których szedłem przez ogrody,

I upłynęło tyle wody,

Jakby to była ludzka krew.

Die Kategorie der Zeit spielt hier ebenfalls eine grundlegende Rolle und zeigt sich bereits im ersten Vers: „Od tych dni dzieli mnie już wiele drzew”. Seit dem Moment, als die Gärtner, die damals Soldaten waren, anfingen, mit ihren Waffen zu schießen sind viele Bäume gewachsen. „Od tych dni dzieli mnie już wiele drzew" - diese Zeile kann man als Rechtfertigung für die umgekehrte Reihenfolge verstehen, in der die Geschehnisse geschildert werden. Die Reihenfolge ergibt sich aus der Retrospektive des lyrischen „Ich”. Zuerst wird thematisiert, was dem lyrischen „Ich” zeitlich nähersteht und erst später das, was vor längerer Zeit geschah. In Mój wiek äußert Wat die Überzeugung, man müsse Literatur rückwärts schreiben ${ }^{27}$. Der Begriff der Zeit ist auch in dem Phrasem „upłynęło tyle wody” versteckt. Die Thematik dieser Strophe bezieht sich also auf den Krieg, bzw. auf die Weltkriege.

Wie bereits erwähnt, ist die erste Strophe inhaltlich geteilt. In der ganzheitlichen Auffassung stellt sie eine Opposition, sowie eine andere Zeitdimension im Vergleich zu der letzten Strophe dar. Diese wiederum verlegt - abgesehen von der großen Anzahl an Bildern - die Handlung des Textes in einen anderen, präzisierten Raum, in die Sahara.

\section{Ästhetisches Warum}

Im diesen Kapitel soll es um die Frage nach den poetischen Verfahren, Äquivalenzbeziehungen und nach dem Sinn des Gedichts gehen.

${ }^{27}$ Vgl. A. Wat, Mój wiek. Pamiętnik mówiony, przedm. C. Miłosz, do druku przygotowała L. Ciołkosz, London 1977. 
Der erste Vers des Gedichtes zeigt den Park ${ }^{28}$ als Arbeitsstelle der Gärtner. Sowohl das Wort „Garten”, als auch andere semantisch ähnliche Bezeichnungen dieses Ortes, wie z.B. „park”, „sad” „Obstgarten” begegnen dem Leser auch während der weiteren Lektüre des Textes. Sie bilden eine Einheit, innerhalb derer man Unterschiede thematisieren kann. Das Kriterium der Oberfläche kann der Maßstab des Unterschiedes sein. Der Garten ist ein ex-situ Schutzort (ein Platz außerhalb des Ursprungsortes). Ein Park ist ein öffentlicher, gestalteter, grüner Stadtraum, der mit Spaziergängen, Sport und aktiver Erholung assoziiert wird und einer großen Anzahl an Menschen zur Verfügung steht. Im Garten kann man sich dem otium hingeben, einer eher intellektuellen Erholung. Man kann sich der geistigen Entwicklung widmen, die von dem nahen Kontakt mit der Pflanzenwelt unterstützt werden kann. Der Garten ist auch ein geschütztes Objekt, das von einer Mauer (hortus conclusus) oder einer symbolischen Umzäunung umgeben ist, um die Arbeit der Gärtner zu schützen. Gärten wurden meist von Anfang an auf Brachland ${ }^{29}$ angelegt. Sie tragen das „Stigma” des Besitzers.

Auch im ersten Vers wird die Arbeit der Gärtner erwähnt. Sie „mähen Rabatte von Hyazinthen und Orchideen" obwohl diese außergewöhnlich schönen Blumen, die auf Grund der Intensität ihrer Farben selten unbemerkt bleiben, in der Regel der Stolz eines jeden Gärtners sind. Die „Macht der Gewohnheit” lässt sie dies jedoch vergessen. Diese Aussage „taka jest moc nawy$\mathrm{ku}^{\prime 30}$ teilt die erste Strophe in zwei Abschnitte und betont die Automatisierung der Arbeit der Gärtner. Gleichzeitig ist sie eine versteckte Form des Protestes gegen die Standardisierung und Technisierung des Lebens. „Das Mähen” mit Hilfe der „rozpylacze” zeugt von der Modernisierung der Arbeit im Park. Die Funktion, die dem Zerstäuber durch das Prädikat („koszą”) zugegeben wurde, besteht in dem „koszenie”, d.h. in dem Abschneiden, der Zerstörung von Leben. Der Name dieses Werkzeugs deutet jedoch auf eine im Prinzip gegensätzliche Aufgabe: Er deutet vor allem auf das Versprühen von Flüssigkeiten, in erster Linie auf das Versprühen von lebensspendendem Wasser.

Wie bereits weiter oben angemerkt wurde, findet sich in dieser Tätigkeit eine Verbindung zum nächsten Teil der ersten Strophe. „Kropili z lepszej bro-

${ }^{28}$ Paradies ist ein Lehnwort aus dem Altiranischen (avestisch „pairi-daēza” = Umwallung, umzäunter Park) Hiervon sind abgeleitet spät babyl. „pardisu” und herb. „pardĕs” (Hohelied 4, 13; Prediger 2, 5 „Lustgarten”; Nehemia 2, 8 „Forst”) sowie griech. $\pi \alpha \rho a ́ \delta \varepsilon เ \sigma o \varsigma$, das zunächst (so bei Xenophon) den Park der Persischen Könige bezeichnet, aber durch die LXX (Gen. 2, 8.15 ) in die religiöse Sphäre erhoben wurde (Vulg. „paradisus”).

Die Religion in Geschichte und Gegenwart. Handwörterbuch für Theologie und Religionswissenschaft, Dritte Auflage, Fünfter Band, Tübingen 1961, s. 95. [Schlusswort:] Paradies. I. Religionsgeschichtlich. [Autor:] F.M.Th. de Liagre Böhl.

${ }_{29}$ Vgl. M. Battistini, op. cit., s. 252.

30 Auf Deutsch: „das ist die Macht der Gewohnheit”. 
ni”31 bedeutet, dass auf die gotischen Türme geschossen wurde. Die Bildlichkeit des Textes fußt demnach auf einer Prädikatisierung, die syntaktische Parallelismen erzeugt. „Rozpylaczami kosič” und „z broni kropić” sind ungewöhnliche Verbindungen, denn man kann nicht mit einem Zerstäuber mähen ${ }^{32}$. Bei dieser außergewöhnlichen Wortverbindung handelt es sich um eine Metapher, die zugleich ein Paradoxon ${ }^{33}$ zu sein scheint. Diese paradoxen Metaphern gehört unter anderem zu den avantgardistischen Techniken ${ }^{34}$. Eine genauere Erklärung dieser Technik befindet sich im Kapitel: „Kulturelles Warum”.

In beiden Fällen handelt es sich um eine Art des wiederholten Handelns. Ähnlich wie die Waffe mit Patrone schießt, versprüht der Zerstäuber Wasser. Beide sind dabei gleichermaßen effektiv. Dieser durch die Wiederholung der Funktion der „,rozpylacze” („Zerstäuber”35) und der Schusswaffen genau bestimmte Rhythmus ist vergleichbar mit der gleichen Anzahl der Akzentsilben.

Durch syntaktische Parallelismen entsteht hier eine Äquivalenzbeziehung. Während des Krieges wurden zahlreiche Symbole der Kultur und Religion zerstört, so wie nun dieselben Gärtner die Blumen im Park „töten”, oder eher ihre Personifikationen. Die Hilflosigkeit der Blumen gegenüber dem Mähen der Gärtner wird mit der Hilflosigkeit der Menschen gegenüber den Kriegen, Waffen und dem Schießen verglichen. Hier wird also die vorherige Rolle der Gärtner entdeckt, die in der Vergangenheit Soldaten waren. Die Aussage „Taka jest moc nawyku” hat also einen teilweise ironischen Unterton. Einst benutzten sie unüberlegt ihre Waffen, schossen auf gotische Türme und Türmchen, auf einen heiligen Platz, was durch die Wiederholungen als Polyptoton betont wird. Nun handeln sie nicht anders, indem sie ebenfalls ohne Bedenken die Blumen kupieren.

Bemerkenswert ist die Auswahl der Blumen. Es stellt sich die Frage, ob sie Zufall ist? Was führt den Autor zu dieser Wahl? Die Hyazinthe blüht im Frühling und wird auch mit dieser Jahreszeit identifiziert, also mit der „Wiedergeburt". Sie verkörpert auch das Göttliche (wie in der Hyakinthos-Sage). Die Orchidee ist wiederum eine äußerst elegante Blume, die die klassische Schön-

${ }^{31}$ Vgl.: Fussnote 27.

32 Die Prädikatisierung erzeugt hier die syntaktischen Parallelismen, gleichzeitig aber eine Bildlichkeit. „Rozpylaczami kosič” und „z broni kropić” sind ungewöhnliche Wortverbindungen.

33 Über Paradoxon bei Staff schreibt J. Kwiatkowski, U podstaw liryki Leopolda Staffa, Warszawa 1966, 87-175.

${ }^{34}$ Siehe Fussnote 29.

35 J. Jagodziński, Bunkry na ruinach. Szkice do historii KL Stutthof-Außenlager Pölitz, oprac. P. Knap, Szczecin 2009, s. 115; J. Misiewicz, Zapiski z dni powstania warszawskiego w Szpitalu Wolskim, „Przegląd Lekarski” 1967, nr 1, s. 149; Alija, Holocaust-Zagtada Żydów, oprac. J. Grzesik, Lublin 2011, s. 136; „Zerstäuber”, gemeint als Gerät zum Zerstäuben von Flüssigkeit, ist eine Anspielung auf die Zerstäubung von Zyklon B, mit dem die Opfer in den Konzentrationslagern getötet wurden. 
heit symbolisiert. Diese Blumen sind wehrlos gegen die Aktivität der Gärtner. Mit anderen Worten - das, was göttlich und schön ist, stirbt bei der Begegnung mit der menschlichen Arbeit. Zudem sehen sowohl Hyazinthen, als auch Orchideen wie Türme aus, also es besteht eine relevante Gleichwertigkeit, eine Äquivalenz zwischen ihnen und den gotischen Türmchen aus Steinspitzen, von denen im zweiten Fragment dieser Strophe die Rede ist.

\section{Grządki}

Hiacyntów i storczyków ähnlich wie

\section{w kamienne koronki \\ wież i wieżyczek}

In der so entstandenen Bildlichkeit ist hier durch die Verwendung eines Enjambements und der Genitivform (samt zweier weiterer Genitivformen der Nomen) auch ein syntaktischer Parallelismus entstanden. Die grammatische Form stimmt überein.

Zurück zu der erwähnten Äquivalenz zwischen den zwei Verbformen, die die Arbeit der Gärtner bezeichnen und mit „k” anfangen: „koszą” und „kropili”. Wie bereits gesagt, beziehen sie sich auf die Tätigkeit der Gärtner und im übertragenen Sinne auf den Begriff der „Tötung”, der Zerstörung. Wenn man aber die hauptsächliche Bedeutung dieser Wörter in Betracht zieht, dann wird „kosić” mit dem Wegnehmen des Lebens verbunden, dagegen bedeutet „kropić” das Gegenteil, da es eher mit dem Verschenken des Lebens in Verbindung gebracht werden kann. Obwohl die beiden Verbformen den unvollendeten Aspekt haben, unterscheiden sie sich voneinander durch die Form: „koszą” (sie mähen) steht im Präsens und „kropili” (versprühten, bestreuten) - im Perfekt/Präteritum. Die Opposition zwischen der Gegenwart und der Vergangenheit wird nochmals hervorgehoben. Es entstehen zwei parallele Mikrostrukturen, Mikroräume innerhalb der ersten Strophe. Die gegenwärtige Sanftheit der Gärtner, der die frühere Brutalität gegenübergestellt wird, weist außerdem auf die Komplexität der menschlichen Natur hin. Diese Diskrepanz zwischen dem guten und bösen „Ich” ist auch in der kontrastierenden Verbindung der Wörter „łagodni” (sanfte) - „koszą” (mähen) sichtbar. Wie kann jemand, der „łagodny” ist, mähen - im Sinne von „schießen”? Je nach den Umständen, in diesem Fall während des Krieges, kommt dieses zweite, gegensätzliche „Ich” eines Menschen zum Vorschein. Daraus kann eine Begründung für die Verwendung des Plurals in dem Titel des Gedichtes folgen. Die menschliche Natur ist sehr schwach und geteilt, wie Mikołaj Sęp-Szarzyński in seinem Sonett IV schreibt:

Cóż będę czynił w tak straszliwym boju,

Wątły, niebaczny, rozdwojony w sobie ${ }^{36}$ ?

${ }^{36}$ M. Sęp-Szarzyński, Sonet IV, O wojnie naszej, która wiedziemy z szatanem, światem i ciatem [w:] idem, Rytmy abo wiersze polskie oraz cykl erotyków, Wrocław 1973, s. 10. 
Jeder hat zwei Seiten in sich. Seine Natur besteht aus seinem eigenen "Ich” - welches hier und jetzt existiert - und aus seinem zweiten „Ich” - welches zur Vergangenheit gehört. Die beiden können nie gleichgesetzt werden.

Jetzt zum zweiten Teil der ersten Strophe, mit der die Analyse angefangen hat. Besondere Aufmerksamkeit sollte man den im Text erwähnten Spitzbögen schenken, sowie auch dem fehlenden Subjekt. Die Bedeutung des Wortes „koronka” ist nicht ganz klar. Eine der Bedeutungen ist „Spitze”. Spitze kann als Produkt des Kunsthandwerks einen großen artistischen Wert besitzen. Sie ist ein Flechtwerk von Fäden, das von Löchern durchbrochen ist, so dass schöne Muster entstehen ${ }^{37}$. Sie symbolisiert also die Zartheit, Kunst sowie Gebetsempfindungen. Hier ist sie auch mit der Ornamentik verbunden.

Nicht ohne Grund fehlt hier das Subjekt des Satzes, welches gemeinsam für die ganze erste Strophe gilt, also die Gärtner. Mit Hilfe vom Kontext der Äusserung (eine kontextuelle Ellipse) und der Situation, welche sie enthält (eine situative Ellipse) lässt sich das Subjekt rekonstruieren. Eine Begründung für die Anwendung der elliptischen Auslassung, kann man im kulturellen Teil finden.

Od tych dni dzieli mnie już wiele drzew,

Wśród których szedłem przez ogrody,

I upłynęło tyle wody,

Jakby to była ludzka krew.

Der Vers „I upłynęło tyle wody” soll „upłynęło tyle czasu” lauten (hier wird das Wort „Zeit” durch das Wort „Wasser” ersetzt) - das ist eine Periphrase. Die Zeit wird mit Wasser identifiziert und mit dem Blutvergießen verglichen. Also kommt es durch diesen Vergleich erstens zur Identifizierung des Wassers mit dem Blut, wodurch die Flüssigkeiten eine Einheit bilden. Zweitens kommen die flüssigen Elemente in Konstellation mit dem Unglück vor. „Upłynęło tyle wody” ist eine Redensart, die von Panta rhei kommt ${ }^{38}$. Das ist ein philosophisches Statement über die Zeit. Aus Heraklits Fluss einen Fluss zu machen, in dem Blut fließt, bedeutet, dass aus einer alten Redensart plötzlich wieder etwas ganz Neues hervorgebracht wird. Woda i krew ist eine relativ konventionelle Metapher, die semantisch relevant ist. Zwischen den beiden Wörtern entsteht eine Äquivalenz gegen die Reime bzw. über die Reimwörter hinweg. Deutliche semantische Parallelismen zwischen den Zeilen der Verse

37 „Koronka” ist aber auch eine Form des Gebets, bei dem man eine bestimmte Anzahl verschiedener Gebete wiederholt, wie z.B. beim Barmherzigkeitsrosenkranz.

${ }^{38}$ In der Mitte steht das Wasser durch einen Vergleich in einer Beziehung zum Blut. Die Zeit wird mit diesem Wasser panta rhei mit dem menschlichen Blut geleichgesetzt. Durch einen Vergleich, wodurch diese Räume noch verglichen werden. Dass die Zeit wie Wasser vergeht. Zeit, Wasser, Blut werden miteinander identifiziert. „Alles fließt” ist ein Aphorismus von Heraklit. Heraklit verglich das Sein mit einem Fluss, indem er sagte, niemand könne zweimal in denselben Fluss steigen. 
zeigen, dass sozusagen thematische Äquivalenzen vorhanden sind (aber keine formalen Äquivalenzen über die Lautgestalt). Woda i krew stehen jedoch in positioneller, benachbarter Äquivalenz und das ist auch etwas Formales.

Drzewa - ogrody ist auch ein Paar, das über die Reimwörter hinausgeht. Wenn man mit Hilfe der Botanik argumentiert, gehören die Bäume zu den Gärten. Es kann hier auch eine pragmatische Beziehung ${ }^{39}$ entstehen: „ogród drzew”, d.h. Gärten voller Bäume, was nochmal ihre gegenseitige Zugehörigkeit zeigt. Die Bäume bestimmen jedoch die vertikale Richtung, die Gärten wiederum - senkrechte Räume.

Im ersten Paar der männlichen Reime: drzew - krew steht die semantische Äquivalenz im Vordergrund. Auf den ersten Blick sieht man den Gegensatz zwischen ihnen - das Blut ist eine fließende Substanz, dagegen ist der Baum eine harte, feste, tief verwurzelte Pflanze. Zwischen ihnen besteht aber auch eine pragmatische Beziehung. Wenn von Blut die Rede ist, kann man das Harz meinen, also die in den Bäumen enthaltene Substanz, die eine analoge Bedeutung zu den Blutplättchen hat.

Zahlreiche Verzweigungen in einer Baumkrone, die vom Baumstamm ausgehen, erinnern an das Blutgefäßsystem des Menschen mit seinen Arterien und Venen. Es lässt das Blut aus dem Herzen herausfließen, metaphorisch gesagt, aus dem Stamm des menschlichen Körpers ${ }^{40}$.

Vom ersten Paar der lexikalisch - oder syntaktisch beschränkten Reime: drzew ${ }^{41}-$ krew ausgehend, folgt der Übergang zu einem allgemeineren Bild, zu dem zweiten Reimpaar. Diesmal handelt es sich um einen weiblichen Reim: ogrody - wody. Es gibt zwischen diesen Reimwörtern eine Art Symbiose. Der Garten erneut sich immer wieder dank des Wasserskreislaufs. Auf diese Weise wird eine pragmatische Beziehung zwischen den beiden Begriffen

39 Terminus aus der Vorlesung von Matthias Freise. Vgl. M. Freise, Slawistische Literaturwissenschaft, Tübingen, Narr 2012, s. 17.

${ }^{40}$ Dies assoziiert mit einer Geschichte, auf die sich Jerzy Stempowski im Essay Drzewa (1950, Bäume) berufen hat, nämlich von Dryope (Ovid, Metamorphoses, Buch IX, Vers 324393), die in einen Baum verwandelt wird. Es war eine Strafverwandlung, weil sie für ihren Sohn einen blühenden Zweig vom Lotoskirschenbaum an einem Kratersee pflückt, ohne zu wissen, dass in den Baum sich die Nymphe Lotis verwandelte hatte. Nach Dryopes Tat begann der Baum zu bluten. Vgl. J. Stempowski, Ziemia berneńska, Warszawa 1990, s. 55.

${ }^{41}$ Die kosmische Achse, der Pfeiler der Welt: Der kosmologische Weltenbaum befindet sich mitten in der Erde, in ihrem „Nabel”. Er verbindet drei kosmische Reiche: Der Stamm ist auf der Erde, die Äste reichen bis zum Himmel, und die Wurzeln gehen hinunter in die Hölle. Er ist ein Symbol des Lebens in allen seinen Erscheinungsformen. Das Buch Genesis sagt, dass in der Mitte des Gartens Eden ein Baum des Lebens und ein Baum der Erkenntnis von Gut und Böse gewachsen waren. Aus dem letzteren wurde das Kreuz, auf dem Christ starb, gemacht. Wie das Kreuz, so symbolisieren diese Bäume den Tod und die Auferstehung. Nach dem Winterschlaf wachsen jedes Jahr neue Knospen - und die geistliche Entwicklung. Als ein Phallussymbol personifiziert der Baum die Beziehung des Menschen zur Natur und ihre gegenseitige Abhängigkeit. M. Battistini, op. cit., s. 248. 
skizziert. So wie der Garten sich verwandelt, wird sich auch das Wasser verändern. Das Wasser ist wechselhaft und ein Symbol der Natur. Es ist unmöglich, die Natur vorauszusehen und man kann sie nicht als etwas Dauerhaftes betrachten. Ein Garten dagegen besteht aus einem Gebiet, das wiederum aus dem ganzen Pflanzenwuchs besteht. Dieser Pflanzenwuchs ist dauerhaft und wird vom Menschen kontrolliert. Dieses System vorhersehbar.

Die Zeit, als eine übergeordnete Kategorie, die die Komposition des Textes organisiert, kommt auch am Anfang der letzten Strophe zum Vorschein.

\section{Tymczasem nawodniona Sahara}

(Die inzwischen bewässerte Sahara)

Die Zeitkategorie wird nicht nur im Wort „tymczasem” (tym + czas + em), sondern auch im Ton des ganzen Verses versteckt. Soviel Zeit ist vergangen, dass sogar die Sahara bewässert wurde - was man für die Wirklichkeit nicht behaupten kann. Das künstlerische Hauptmittel ist hier ein hyperbolisches Oxymoron in einer Form, die zu einem Vergleich übergeht. Als größte Wüste in Afrika erfüllt die Sahara keine angemessenen Bedingungen für die Entwicklung des Lebens. Mittlerweile erwacht sie aber zu neuem Leben, unterliegt Verwandlungen. Daraus folgt, dass bisher alles zerstört worden ist. Die Sahara ist symbolisch ein Schlachtfeld, auf dem ein Krieg ausgetragen wurde.

\section{Tymczasem nawodniona Sahara}

Rozkwitła cała jak jeden sad.

Diese Aussage enthält auch räumliche Äquivalenzen zwischen der Sahara und dem „sad”42. Die bewässerte Sahara ist ein Übergang in diese Vergangenheit. Das Gebiet, das nach dem Krieg übriggeblieben ist, sieht aus wie eine Wüste. Mit der Zeit wird sie allerdings wieder erblühen. Die Sahara steht für die Vergangenheit. Sie ist eine Metonymie der Vergangenheit.

Die Frage ist nur, wer für diesen „sad” verantwortlich ist? Wo sind die Gärtner geblieben?

Rozkwitła cała jak jeden sad.

W nocy księżyc podlewa go rosą

I w mgle tancerka tańczy boso,

Wśród kwiatów sama jak kwiat.

We dnie, pod gołym niebem goły,

W słońcu opala się mój brat.

Ale ma trupa oczodoły.

${ }^{42}$ Auf Streuobstwiesen stehen hochstämmige Obstbäume meist unterschiedlichen Alters und unterschiedlicher Arten und Sorten. 
Der neu entstandene Garten wird in der Nacht vom Mond mit Tau begossen: „W nocy księżyc podlewa go rosą”. Hier gibt es eine sogenannte verbale Metapher, weil der Mond, der personifiziert ist, nicht in der Lage ist, etwas mit Wasser zu begießen. „Podlewać” gehört zu den menschlichen Tätigkeiten. Das Motiv des Mondes ist charakteristisch für das Junge Polen. Er bekommt oftmals figurative Qualitäten ${ }^{43}$. In diesem Fall übernimmt der Mond zugleich teilweise die Rolle der Gärtner, die in diesem Abschnitt des Gedichtes fehlen. Dieser neue Garten wird also durch die Metaphorik, durch die Prädikatisierung des Wortes „podlewać” und schließlich durch die Personifikation „księżyc” beschrieben. Die Personifikation ist eine Kategorie der Allegorie. Die Wiederholungen „We dnie, pod gołym niebem goły”, bekommen dank der Verwendung von Polyptota eine onomatopoetische Wirkung.

In diesem neuen Garten sonnt sich nun der Bruder des lyrischen „Ichs”. Er besitzt die Augenhöhlen einer Leiche, liegt nackt und frei unter freiem Himmel. Hier entsteht nicht nur eine Anknüpfung an die Nacktheit der ersten von Gott geschaffenen Menschen, also an die Rückkehr zum ursprünglichen Zustand der Dinge auf der Erde. Darüber hinaus ergibt sich auch eine Zugehörigkeit zwischen dem nackten, freien Bruder und dem freien Himmel, die aus ihrer gemeinsamen Natur entstanden sind und die eine gewisse geschlossene Einheit bilden. Die metaphysische Ebene dieses Fragments geht auf die Beziehung des Menschen zur Natur ein, aber auch auf die Gegenüberstellung der Einsamkeit des Individuums dieser Strophe mit der Gemeinschaft, dem Kollektiv der Gärtner in der ersten Strophe.

Vor dem Leser entfaltet sich das Bild des Parks mit den arbeitenden Gärtnern, den besonderen Blumen. Danach geht die Situation in eine Welt der Phantasie, der Erinnerung über. Diese Bilder beeinflussen also das Verständnis des Raumkonzepts. Der Raum wird mobil und enthält nun zwei Zeitdimensionen, die nebeneinander existieren - mal Gegenwart, mal Vergangenheit. Diese Bildsymbolik ruft nicht nur diese zeitliche Dimension, sondern auch die räumliche hervor. Das führt wiederum zur Entstehung der zeitlichen und räumlichen Äquivalenz, die von dieser kühnen Metapher ${ }^{44}$ ernannt und die durch syntaktische Parallelismen verstärkt wird. Die Gärtner sind also „Reisende”, die einen Spaziergang durch Zeit und Raum machen.

In diesem Gedicht dominieren die Polyptota: „Gotyckich wież i wieżyczek; w mgle tancerka tańczy boso; wśród kwiatów sama jak kwiat, pod gołym niebem goły". Aber es lassen sich auch syntaktische Parallelismen beobachten: „W nocy”, „w mgle”, „we dnie”, „w słońcu”. Im Abschluss entsteht ein Kon-

${ }^{43}$ Die Nacht ist mit Wasser und Unterwelt verbunden, denn dort befinden sich die Keime aller Lebensweisen. Vgl. M. Battistini, op. cit., s. 68.

${ }^{44}$ Z.B. „Rozpylaczami koszą grządki”. Die Raumäquivalenzen werden durch diese kühne Metaphorik hervorgerufen und syntaktisch durch die Parallelismen unterstützt. 
trast, weil dem hellen, sonnigen Naturraum die Augen einer Leiche gegenübergestellt werden.

W słońcu opala się mój brat.

Ale ma trupa oczodoły.

\section{Kulturelles Warum}

$\mathrm{Zu}$ einer vollständigen Untersuchung des Gedichts gehört auch die Untersuchung des kulturellen Warums. Diese beschäftigt sich mit der Frage nach den intertextuellen Beziehungen, die hier in dem schon erwähnten Modernismus-Kontext zu finden sind, in der Lautmalerei und Metaphorik. Dieser Kontext unterscheidet sich von der Art der Kreation der Bilder in der ersten Strophe. Im ersten Abschnitt des Gedichtes gab es eine sichtbare Anknüpfung an die Avantgarde oder, genauer gesagt, an das Programm der Krakauer Avantgarde, d.h. an Tadeusz Peipers Theorie der Metapher ${ }^{45}$. Laut Peiper steckt der Sinn der Poetik in der Kürze und Kraft der Metapher, in der Kondensierung der Wortbedeutung. Die Metapher entspreche am besten der von der zeitgenössischen Zivilisation beeinflussten Art des Empfindens und ermögliche die maximale „Ökonomie” des Ausdrucks. Dieser Theorie zufolge ist das grundsätzliche Element des Gedichtes ein Satz, der eine mehrfache Metapher enthält. Wichtig ist die maximale Verdichtung der Bedeutung (eine Kondensierung: ein Wort kann mehrere nötige Assoziationen hervorrufen, wie bei Staff die Wörter „koszą” bzw. „kropili”), eine Anhäufung von vielen einzigartigen Metaphern und das Streben nach Kürze. Der Dichter ist hier also kein Priester, kein sorgloser Flattergeist. Stattdessen ist er ein seriöser Handwerker. Der Kreationismus in der Poesie dieses Autors beruht auf der Überzeugung, dass ein Dichter die Welt in Wortmaterial darstellt ${ }^{46}$.

Beispielsweise lassen sich in der Dichtung von Przyboś viele typische, einzigartige Metaphern ${ }^{47}$ finden, die in der Genauigkeit und Konkretheit ihrer Bildkreation überraschen. Er bedient sich öfter der Ellipse, die der ökonomischen Disziplin der Gedichte gut angepasst ist. Ellipsen entstehen durch das Auslassen einzelner Wörter oder ganzer Satzteile, so dass die Aussage häufig erst nach der Lektüre des gesamten Textes verständlich wird. Für seine früheren Texte ist auch eine Faszination für Technik charakteristisch. Dies kann man an der spezifischen Auswahl von Wörtern sehen, die aus Bereichen wie

${ }^{45}$ Vgl. T. Peiper, Tędy. Nowe usta, przedm. S. Jaworski, Kraków 1972, s. 54-61.

${ }^{46}$ Vgl. G. Gazda, Stownik europejskich kierunków i grup literackich XX wieku, Warszawa 2009, s. 55-56.

${ }^{47}$ R. Skręt, Julian Przyboś. Utwory poetyckie, Kraków 1984, s. 148. „Kamieniuje tę przestrzeń niewybuchły huk skał./ To - wrzask wody obdzieranej siklawą z łożyska i/ Gromobicie ciszy” 
der Physik oder Geometrie stammen, wie z.B. „rozpylacz” bei Staff. Alle oben genannten Merkmale, die in der Avantgarde dominieren, sind in der ersten Strophe des Gedichts von Staff zu finden, was bereits im ästhetischen Teil dieser Arbeit behandelt wurde.

Im letzten Abschnitt des Werkes drängt sich wiederum der Jungpolnische Dichter in den Vordergrund. Man sieht ein ziemlich konventionelles Bild des Gartens, in dem eine Tänzerin barfuß im Nebel tanzt: „w mgle tancerka tańczy boso”. Durch das Todesmotiv, das in den Augenhöhlen der Leiche „trupa oczodoły” Ausdruck findet, wird sie etwas verfremdet. Das alles klingt aber sehr idyllisch. Im Gegenteil zu der ersten Strophe, die von Dynamik dominiert ist (der Park, die mähenden Gärtner, die sich ständig bewegen - auch die gotischen Türme und Türmchen), ist die dritte Strophe eher mit statischen Bildern versehen. Als Beispiel kann der Bruder des lyrischen „Ichs” herangezogen werden, der sich höchstwahrscheinlich sonnt.

In den neoromantischen Gedichten ${ }^{48}$ ist ebenfalls die Stimmung von Bedeutung. Es ist eine unklare und nicht ganz definierte Stimmung, die aber einen unterbewussten wahrnehmbaren Zustand der Seele darstellt, den man sich durch einen durchsichtigen Schleier anschaut. Diesen Zustand markieren Verträumtheit, Traurigkeit, melancholische Grübelei und Kontemplation über Ästhetik und Gefühle. Diese Stimmung wurde zu einer natürlichen Art des modernistischen Erlebens der Wirklichkeit ${ }^{49}$. Sie findet sich in den ersten zwei Worten jeden Verses der letzten Strophe:

Tymczasem nawodniona Sahara

Rozkwitła cała jak jeden sad.

W nocy księżyc podlewa go rosą

I w mgle tancerka tańczy boso,

Wśród kwiatów sama jak kwiat.

We dnie, pod gołym niebem goły,

W słońcu opala się mój brat.

Ale ma trupa oczodoły.

Die meisten dieser Verse fangen mit einer Anapher an, wodurch der romantisch-melodische Aspekt der Stimmung in dieser Strophe betont wird.

Der neoromantische Ästhetizismus wurde zum Prototyp des Expressionismus, vor allem in seiner Tendenz zur Hyperbolisierung des Ausdrucks (der bewussten Übertreibung) und der Anwendung von einem Wortschatz und einer Phraseologie mit superlativem Bedeutungsaspekt. Dingen und Gegenständen, mentalen Zuständen und Naturerscheinungen hat man eine ungewöhnliche Größe, Kondensierung und Intensität zugesprochen. Dieses Be-

48 Stownik terminów literackich, red. J. Sławiński, Wrocław 2002, s. 340.

${ }^{49}$ Vgl. A. Hutnikiewicz, op. cit., s. 92. 
dürfnis nach Expression verlangt von dem Dichter eine ständige Suche und im Rahmen dieser Suche nach „neuen Formen” greift er nicht nur nach Errungenschaften der europäischen Poesie, sondern auch nach Einflüssen aus den exotischen und orientalischen Literaturen. Formale und stilistische Strukturen, sowie Versstrukturen werden in die Literatur der jeweiligen Muttersprache eingeführt ${ }^{50}$. In dem Beispiel des Gedichtes von Staff wird die Sahara genannt. Es kommt nicht nur zu einer Kollision der Kulturen, sondern auch zu einer Art Kulturanpassung. Es wird ein Prozess ausgelöst, der die Gesamtheit der gesellschaftlichen und psychischen Veränderungen bezeichnet, die ein Mensch durchmacht, wenn er sich von seiner Kulturgruppe trennt und versucht, sich dem Leben in einer anderen Kultur anzupassen. Bei Staff wird den Gärtnern aus dem Titel erstens der Bruder des lyrischen „Ichs” gegenübergestellt, der sich in einem neuen kulturellen Raum befindet. Seine ausgehöhlten Augen brechen die romantische Idylle des Raumes und verweisen auf seine Zugehörigkeit zu einer früheren „Epoche”. Zweitens wird ihnen ebenfalls das lyrische „Ich” selbst gegenübergestellt, wenn es sagt:

Od tych dni dzieli mnie już wiele drzew,

Wśród których szedłem przez ogrody.

Es sieht sich selbst in einem völlig anderen Naturraum. Es hat sich nicht nur der Ort verändert, sondern vor allem die Mentalität, die Einstellung.

Auch wenn etwas Neues entsteht, das schon in der ersten Strophe einbezogen wurde, so bleibt immer noch der Bezug zum Jungen Polen erhalten. Darin liegt die Besonderheit des Topos der Gärtner bei Staff - ein Verweis auf die Poetik des Jungen Polens und damit die Forderung nach einer Rückkehr zu den Wurzeln. Staff kehrt wieder zu der alten Poetik zurück.

Der Verweis auf die Vergangenheit steht ebenfalls in Verbindung mit den Erinnerungen, die wiederum auf Freud verweisen. Im Verlauf der Zeit behalten sie ihre Intensität. Wie R. Scott Bakker einmal gesagt hat: „Einige Ereignisse hatten sich so tief in uns geprägt, dass sie erst viel später bedeutsamer wurden" ${ }^{51}$. Die Konfrontation der Gärtner mit dem Zerstäuber und den Blumen führt zu einer Begegnung mit verdrängten Erinnerungen, die aus dem Alltagsleben bzw. aus dem Bewusstsein weitgehend entfernt wurden. Nach wie vor sind diese jedoch sehr präsent. Dieser Prozess zeigt Gemeinsamkeiten mit der Psychoanalyse von Freud - die Wiederkehr des Verdrängten. Die Gärtner sind nicht nur dazu gezwungen, ihre Vernunft und Gedanken räumlich und zeitlich zuzuordnen, sondern auch das unangenehme Geschehen aus der Vergangenheit wieder auferstehen zu lassen. Einige Erinnerungen sind von dem restlichen psychischen Leben isoliert. „Koszenie” ist eine Metapher

${ }^{50}$ Vgl. ibidem, s. 93.

${ }^{51}$ M. Wojdakowska, Mata księga cytatów część 1, Warszawa 2003, s. 31. 
für das „strzelanie”, was eine quälende Erinnerung darstellt. Es ist das, was Freud als Verdrängung bezeichnet. „Rozpylacz” und Blumen - diese Gegenstände erwecken die unliebsame Vergangenheit, die Einfluss auf die jetzige psychische Lage der Hauptpersonen hat. Als auslösender Faktor ist das ein Impuls, der zur Aufklärung dessen führt, was verdrängt wurde ${ }^{52}$. „Rozpylacz” stellt die Erinnerung an das „strzelanie” wieder her. Der Zerstäuber und die Blumen erlauben es, die Aktivität des psychischen Unterbewusstseins zu erkennen. Sie setzten einen Automatismus in Gang, der unliebsame Erinnerungen wachmacht und sie mit dem Geschehen verbindet.

\section{Fazit}

Obwohl hier im Mittelpunkt eine möglichst textnahe Interpretation stand, hat sich der Topos des „Gärtners” als ein wichtiges Element beim Verfassen des Textes erwiesen und bildet damit ein wichtiges Instrument der Analyse. Dies gilt insbesondere für Epochen und Autoren, die stark von der Tradition geprägt sind wie Neoklassizisten, für deren Verständnis eine gründliche Kenntnis des Topos eine indispensable Vorbedingung ist. Staff gehörte zu dem Kreis der Neoklassizisten und sein Gedicht Ogrodnicy kann als Text des Neoklassizismus gelesen werden. Karol Irzykowski verweist in seiner Rezension mit dem Titel Nowe poezje Staffa auf den Klassizismus als wichtiges Merkmal der Kreativität eines Autors:

Staff w pokoleniu zarania XX wieku jest odnowionym typem pseudoklasycyzmu. Poczucie formy i kapłańska dbałość o formę, przewaga logiki nad przypadkowością, fundament intelektualny, rozważna świadomość nad architekturą poezji, wreszcie rozmiłowanie smaku nad pięknem antycznym - wszystkie cechy jego poezji nawodzą wspomnienie poetów końca XVIII stulecia ${ }^{53}$.

Es gibt noch, vor dem Hintergrund des „Gärtner-Topos” untersucht werden sollen, zwei weitere Gedichte: Ogrodnicy (1964) von Kazimierz Wierzyński und Ogrodnik (2001) von Czesław Miłosz. Die Texte deuten schon durch ihren ähnlichen Titel auf ihre Zugehörigkeit zu einem bestimmten Topos hin, nämlich dem des Gärtners. Diese Gedichte bestätigt die Kontinuität der Verwendung von dem Topos des „Gärtners”. Dadurch, dass dieser Topos „wan-

${ }^{52}$ Man kann diese Metapher der Verdrängung auch als eine Verdichtung sehen. Sowohl Verdichtung als auch Verschiebung (Metonymie) stammen von Jakobson. Sie wurden aber auf S. Freuds Traumdeutung von Jacques Lacan aufgebaut. Jakobsons These lautet: „Das Unbewusste ist strukturiert wie eine Sprache".

Vgl. W. Schönau, Einführung in die psychoanalytische Literaturwissenschaft, Stuttgart 1991, s. 154-164; G. Pagel, Lacan zur Einführung, Hamburg 1989.

53 „Kurier Warszawski” 1908, nr 198. 
dert”, erhält er damit die verschiedenen Zeit und Raumkoordinaten. In Erinnerung kann an dieser Stelle der Versuch der Erweiterung des Toposbegriff von Rainer Grübel gerufen werden. Seine topische Modellierung findet die Verwendung bei allen drei Gedichten. Diatopisch vs. Diachronisch wurde das Gedicht Ogrodnicy von Wierzyński angeordnet; Synchronisch vs. isotopisch Ogrodnicy von Staff; Panchronisch vs. pantopisch - Ogrodnik von Miłosz.

Man kann sich fragen, in welchem Zusammenhang die drei Dichter miteinander stehen. Ob der Topos des „Gärtners” im Laufe der Zeit viele Veränderungen erfährt, und welche unterschiedlichen Formen unter der Feder dieser drei Schriftsteller annimmt?

Es ist aber herauszustellen, dass sie die gleiche Botschaft vermitteln, dass der Begriff „Topos” so lebendig wie ein Garten ist, der nach jeder Zerstörung, auch von dem Teufel, zu neuem Leben erwacht. Der Unterschied liegt lediglich darin, dass der Garten im Gegenteil zum Topos zerstört werden kann.

Wir alle stammen aus einem Garten, wie Jonasz Kofta sang. Die menschliche Geschichte, das menschliche Leben, und damit auch ein Leben des Gärtners, beginnen wie diese Analyse in dem verlorenen Paradies und enden auch da.

Man sagt, dass ein Mensch am Ende seines Lebens über sein Leben reflektiert. Man mag nun annehmen, dass Staff, Wierzyński und Miłosz hinsichtlich ihres Lebensabends über das Leben im Allgemeinen mithilfe des Topos des Gärtners reflektierten ${ }^{54}$ - vielleicht mit dem Bild, das Kofta beschreibt, im Sinn.

A o szyby deszcz dzwoni, deszcz dzwoni jesienny...

\section{Bibliografia}

Alija, Holocaust - Zagtada Żydów, oprac. J. Grzesik, Lublin 2011.

Battistini M., Symbole i alegorie, tłum. K. Dyjas, Warszawa 2005.

Boratyński A., Wspominam Leopolda Staffa, http://www.skarzysko24.pl/content/ view/748/109/, abgerufen am 24.08.2011.

Die Religion in Geschichte und Gegenwart. Handwörterbuch für Theologie und Religionswissenschaft, Dritte Auflage, Fünfter Band, Tübingen 1961. [Schlusswort:] Paradies. I. Religionsgeschichtlich. [Autor:] F.M.Th. de Liagre Böhl.

Dłuska M., Prozodia języka polskiego, Kraków 1947.

Freise M., Slawistische Literaturwissenschaft, Tübingen, Narr 2012.

${ }^{54}$ In Vorahnung des baldigen Todes entstanden oder gar gleichzeitig in Bezug auf den Tod anderer Dichter und deren Gärtnergedichten: 10 Jahre jünger ist das Werk von Kazimierz Wierzyński. Er war 70 Jahre alt und war noch fünf Jahre am Leben nach der Veröffentlichung dieses Gedichts. Der „jüngste Brüder” des 90-jährigen Autors erschien im Jahr 2001, drei Jahre vor Miłosz Tod. 
Freise M., Vier Weisen, nach dem Text zu fragen [in:] Finis coronat opus. Festschrift für Walter Kroll zum 65. Geburtstag, Göttingen 2006.

Gazda G., Stownik europejskich kierunków i grup literackich XX wieku, Warszawa 2009.

Grübel R., Zwischen „Leier” und „Trommel”, zur Funktion zweier Topoi im Wechselverhältnis von Struktur und Selbstverständnis russischer Avantgardistischer Lyrik, „Wiener Slawistischer Almanach”, red. A.A. Hansen-Löve, Bd. 2, Wien 1978.

Hutnikiewicz A., Mtoda Polska, Warszawa 2004.

Jagodziński J., Bunkry na ruinach. Szkice do historii KL Stutthof-Außenlager Pölitz, oprac. P. Knap, Szczecin 2009.

Kofta J., Pamiętajcie o ogrodach, http://www.tekstowo.pl/piosenka,jonasz_kofta, pamietajcie_o_ogrodach.html, abgerufen am 24.07.2011.

„Kurier Warszawski” 1908, nr 198.

Kwiatkowski J., Literatura Dwudziestolecia, Warszawa 1990.

Kwiatkowski J., U podstaw liryki Leopolda Staffa, Warszawa 1966.

Maciejewska I., Leopold Staff. Warszawski okres twórczości, Warszawa 1973.

Misiewicz J., Zapiski z dni powstania warszawskiego w Szpitalu Wolskim, „Przegląd Lekarski” 1967, nr 1.

Pagel G., Lacan zur Einführung, Hamburg 1989.

Peiper T., Tędy. Nowe usta, przedm. S. Jaworski, Kraków 1972.

Schönau W., Einführung in die psychoanalytische Literaturwissenschaft, Stuttgart 1991.

Sęp-Szarzyński M., Sonet IV, O wojnie naszej, którą wiedziemy z szatanem, światem i ciatem [w:] idem, Rytmy abo wiersze polskie oraz cykl erotyków, Wrocław 1973.

Skręt R., Julian Przybos. Utwory poetyckie, Kraków 1984.

Stownik języka polskiego, red. J. Karłowicz, A.A. Kryński, W. Niedźwiedzki, t. 2, Warszawa 1900-1927.

Stownik języka polskiego, red. M. Szymczak, Warszawa 1978.

Stownik jezyka polskiego, red. W. Doroszewski, t. 3, Warszawa 1961.

Stownik terminów literackich, red. J. Sławiński, Wrocław 2002.

Staff L., Poezje zebrane, t. 1, Warszawa 1967.

Staff L., Wybór poezji, Wrocław 1970.

Stempowski J., Ziemia berneńska, Warszawa 1990.

Szypowska M., Leopold Staff. Wiersze zebrane, t. 5, Warszawa 1955.

Wat A., Mój wiek. Pamiętnik mówiony, przedm. C. Miłosz, do druku przygotowała L. Ciołkosz, London 1977.

Wojdakowska M., Mata ksiega cytatów część 1, Warszawa 2003.

Wysocki A., Poeta nescit finire. U Leopolda Staffa (w 75-lecie urodzin), „Życie Warszawy” 1953, nr 273. 